\title{
Medullary Thyroid Carcinoma pN1b TNM
} Finding 88

National Cancer Institute

\section{Source}

National Cancer Institute. Medullary Thyroid Carcinoma pN1b TNM Finding v8. NCI

Thesaurus. Code C141040.

Metastasis to unilateral, bilateral, or contralateral lateral neck lymph nodes levels I, II, III, IV, or V or retropharyngeal lymph nodes. (from AJCC 8th Ed.) 